\title{
DEGREE-INVERTING INVOLUTION ON FULL SQUARE AND TRIANGULAR MATRICES
}

\author{
LAIS S. DA FONSECA, EDNEI A. SANTULO JR., AND FELIPE Y. YASUMURA
}

Abstract. In this short note, we classify the degree-inverting involution on the full square and triangular matrices.

\section{INTRODUCTION}

Graded rings appear naturally in several branches of Mathematics and Physics. For instance, one can construct a grading from a filtered algebra, a local valuation ring, a polynomial ring, an automorphism of finite order of an algebra, a finitedimensional Lie algebra over an algebraically closed field of characteristic zero, etc.

Recall that a $G$-grading on an algebra $\mathcal{A}$ is a vector space decomposition $\mathcal{A}=$ $\bigoplus_{g \in G} \mathcal{A}_{g}$ such that $\mathcal{A}_{g} \mathcal{A}_{h} \subseteq \mathcal{A}_{g h}$, for all $g, h \in G$. Some graded algebras are endowed also with a graded involution, in the following sense: $\psi$ is an involution of $\mathcal{A}$, and $\psi\left(\mathcal{A}_{g}\right) \subseteq \mathcal{A}_{g^{-1}}$, for any $g \in G$. Examples include: the usual transposition of square matrices with any good grading, the usual involution on Leavitt path algebras endowed with their usual grading, etc. It is worth mentioning that, in a recent work [13], the authors proved that the graded involution enriches the structure of the Graded Grothendieck group of a graded ring. Thus, understanding gradings and graded involution on a given algebra seems to be an interesting problem. From now on, we will refer the graded involution as degree-inverting involution.

On the other hand, in [3, the authors call a graded involution an involution satisfying $\psi\left(\mathcal{A}_{g}\right) \subseteq \mathcal{A}_{g}$, for all $g \in G$. They proved that the degree-preserving involutions are fundamental to give a description of group gradings on some simple Lie algebras, a question raised by Patera and Zassenhaus [17. After the contribution of several authors, the classification of the degree-presernving involutions on matrix algebras, and the gradings on simple Lie algebras are essentially complete 1, 2, 3, 4, 8, (among others), see also the monograph [9]. Thus, degree-preserving involution is an essential tool as well.

In this paper, using the ideas of the degree-preserving case [10, 1] (see also [9]), we classify degree-inverting involutions on matrix algebras and on upper triangular matrices, improving the results of 11 .

This paper is divided as follows: we include a few preliminary theory in Section2 Then, we provide partial results for degree-inverting involution on graded division algebras (Section 3). Next, we copy the theory presented in [9, Section 2.4] (see also the paper by Elduque [10]) to study the matrix algebra case in Section 4. Finally, in Section 5, we obtain results for the upper triangular matrices case.

L. S. da Fonseca was financed by the Coordenação de Aperfeiçoamento de Pessoal de Nível Superior - Brasil (CAPES) - Finance Code 001.

F. Y. Yasumura was financed by the Coordenação de Aperfeiçoamento de Pessoal de Nível Superior - Brasil (CAPES) - Finance Code 001. 


\section{Preliminaries}

2.1. Graded Algebras. We shall work with graded algebras rather than graded rings, as follows. Let $G$ be any group. We say that an algebra $\mathcal{A}$ is $G$-graded if there exists a vector-space decomposition $\mathcal{A}=\bigoplus_{g \in G} \mathcal{A}_{g}$ such that $\mathcal{A}_{g} \mathcal{A}_{h} \subseteq \mathcal{A}_{g h}$, for all $g, h \in G$. The subspace $\mathcal{A}_{g}$ is called homogeneous component of degree $g$. A nonzero element $x \in \mathcal{A}_{g}$ is called a homogeneous element of degree $g$. We denote $\operatorname{deg} x=g$.

A map $f: \mathcal{A} \rightarrow \mathcal{B}$ between two $G$-graded algebras is called a graded homomorphism if $f$ is an algebra homomorphism, and $f\left(\mathcal{A}_{g}\right) \subseteq \mathcal{B}_{g}$ for all $g \in G$. If, moreover, $f$ is an isomorphism, then $f$ is called a graded isomorphism; in this case, $\mathcal{A}$ and $\mathcal{B}$ are said to be isomorphic.

A graded division algebra is an associative algebra $\mathcal{D}$ with 1 , where each nonzero homogeneous element $x \in \mathcal{D}$ is invertible.

Now let $\mathcal{R}=M_{n}(\mathbb{F})$ be a matrix algebra endowed with a $G$-grading. Then the graded version of the Density Theorem tells us that we can find a graded division algebra $\mathcal{D}, \operatorname{dim} \mathcal{D}=\ell^{2}$, and a sequence $\left(g_{1}, \ldots, g_{m}\right)$, such that $\mathcal{R} \cong M_{m}(\mathbb{F}) \otimes \mathcal{D}$, where the grading is given by

$$
\operatorname{deg} e_{i j} \otimes d=g_{i} \operatorname{deg}(d) g_{j}^{-1}, \quad d \in \mathcal{D} \text { homogeneous. }
$$

Let $\mathcal{A}=\bigoplus_{g \in G} \mathcal{A}_{g}$ be a $G$-graded algebra. We say that $V$ is a $G$-graded right $\mathcal{A}$-module if $V$ is a right $\mathcal{A}$-module and there exists a vector space decomposition $V=\bigoplus_{g \in G} V_{g}$ such that $V_{h} \mathcal{A}_{g} \subseteq V_{h g}$, for all $g, h \in G$. Similarly we define the notion of graded left modules.

Given two $G$-graded right $\mathcal{A}$-modules $V$ and $W$, we say that $f: V \rightarrow W$ is a graded map of degree $g$ if $f$ is an $\mathcal{A}$-homomorphism, and $f\left(V_{h}\right) \subseteq W_{g h}$, for all $h \in G$. The graded maps of degree 1 are also known as graded $\mathcal{A}$-homomorphism. We denote $\operatorname{Hom}_{g}(V, W)$ the set of all graded maps of degree $g$; and let $\operatorname{Hom}_{\mathrm{gr}}(V, W)=$ $\bigoplus_{g \in G} \operatorname{Hom}_{g}(V, W)$. If $V$ and $W$ are finite-dimensional, then we have $\operatorname{Hom}(V, W)=$ $\operatorname{Hom}_{\mathrm{gr}}(V, W)$, so $\operatorname{Hom}(V, W)$ gets a vector-space grading.

Now, let $\mathcal{D}$ be a finite-dimensional graded division algebra, and let $V$ be a finitedimensional $G$-graded right $\mathcal{D}$-module. Then $\mathcal{R}=\operatorname{End}_{\mathcal{D}}(V)=\operatorname{Hom}(V, V)$ is a $G$-graded algebra isomorphic to a matrix algebra. Moreover, $V$ is a $G$-graded left $\mathcal{R}$-module.

Finally, we provide a precise definition of the following:

Definition. Let $\mathcal{A}=\bigoplus_{g \in G} \mathcal{A}_{g}$ be a $G$-graded algebra. An involution $\psi$ on $\mathcal{A}$ is a degree-inverting involution if $\psi\left(\mathcal{A}_{g}\right) \subseteq \mathcal{A}_{g^{-1}}$, for all $g \in G$.

In this paper, involution will mean a first kind involution, that is, a $C(\mathcal{A})$-linear map, where $C(\mathcal{A})$ is the center of $\mathcal{A}$.

2.2. Factor sets. Let $T$ be a finite group. A map $\sigma: T \times T \rightarrow \mathbb{F}^{\times}$, where $\mathbb{F}^{\times}$is the set of invertible elements of $\mathbb{F}$, is called a 2-cocycle or a factor set if

$$
\sigma(u, v) \sigma(u v, w)=\sigma(u, v w) \sigma(v, w), \quad \forall u, v, w \in T
$$

These objects are interesting and intensively studied in the context of cohomology of groups (see, for instance, [15, 16]). However, we do not need such generalities, and we limit ourselves within the theory we will need. 
Denote by $Z^{2}\left(T, \mathbb{F}^{\times}\right)$the set of all factor sets. Since $\mathbb{F}^{\times}$is commutative with respect to the product, the $Z^{2}\left(T, \mathbb{F}^{\times}\right)$acquires a natural structure of abelian group, by point-wise product.

We can construct algebras from factor sets. Given an arbitrary map $\sigma: T \times T \rightarrow$ $\mathbb{F}^{\times}$denote by $\mathbb{F}^{\sigma} T$ the following algebra: $\mathbb{F}^{\sigma} T$ has a basis $\left\{X_{u} \mid u \in T\right\}$, and the product is defined by $X_{u} X_{v}=\sigma(u, v) X_{u v}$. Note that $\mathbb{F}^{\sigma} T$ is associative if and only if $\sigma \in Z^{2}\left(T, \mathbb{F}^{\times}\right)$. For instance, if $\sigma=1$ (the constant function), then $\mathbb{F}^{\sigma} T$ is the group algebra of $T$. Next, we investigate the isomorphism classes of algebras given by factor sets.

For any arbitrary map $\lambda: T \rightarrow \mathbb{F}^{\times}$, we obtain a factor set $\delta \lambda$ by the formula

$$
\delta \lambda(u v):=\frac{\lambda(u) \lambda(v)}{\lambda(u v)} .
$$

Since $\delta\left(\lambda_{1} \lambda_{2}\right)=\delta \lambda_{1} \delta \lambda_{2}, B^{2}\left(T, \mathbb{F}^{\times}\right):=\left\{\delta \lambda \mid \lambda: T \rightarrow \mathbb{F}^{\times}\right\}$is a subgroup of $Z^{2}\left(T, \mathbb{F}^{\times}\right)$. We denote the quotient by $H^{2}\left(T, \mathbb{F}^{\times}\right)=Z^{2}\left(T, \mathbb{F}^{\times}\right) / B^{2}\left(T, \mathbb{F}^{\times}\right)$, and call it the second cohomology group of $T$. Given $\sigma \in Z^{2}\left(T, \mathbb{F}^{\times}\right)$, we denote by $[\sigma]$ the element $\sigma B^{2}\left(T, \mathbb{F}^{\times}\right)$in $H^{2}\left(T, \mathbb{F}^{\times}\right)$.

Lemma 1 ([15, Chapter 2, Lemma 1.1]). Let $\sigma_{1}, \sigma_{2} \in Z^{2}\left(T, \mathbb{F}^{\times}\right)$. Then $\mathbb{F}^{\sigma_{1}} T \cong$ $\mathbb{F}^{\sigma_{2}} T$ if and only if $\left[\sigma_{1}\right]=\left[\sigma_{2}\right]$.

The following is an easy manipulation:

Lemma 2. Let $[\sigma] \in H^{2}\left(T, \mathbb{F}^{\times}\right)$. Then, there exists $\sigma^{\prime} \in[\sigma]$ such that $\sigma^{\prime}(u, 1)=$ $\sigma^{\prime}(1, u)=1$, for all $u \in T$.

Hence, combining the two previous result, given $\mathbb{F}^{\sigma} T$, we can assume that $\sigma(u, 1)=\sigma(1, u)=1$, for all $u \in T$.

Finally, it is worth mentioning that, if char $\mathbb{F}$ does not divide $|T|$, then $\mathbb{F}^{\sigma} T$ is semiprimitive (that is, its Jacobson radical is zero).

2.3. Graded division algebras. Graded division algebras have a nice description when the base field is algebraically closed. Assume that $\mathbb{F}$ is algebraically closed and let $\mathcal{D}=\bigoplus_{g \in G} \mathcal{D}_{g}$ be a finite-dimensional graded division algebra over $\mathbb{F}$. Let $T=\left\{g \in G \mid \mathcal{D}_{g} \neq 0\right\}$ be its support. Then it is easy to see that $T$ is a subgroup of $G$. We use multiplicative notation for the product of $T$, and denote by 1 its neutral element.

Moreover, $\mathcal{D}_{1} \supseteq \mathbb{F}$ is a division algebra. So $\mathcal{D}_{1}=\mathbb{F}$, since $\mathbb{F}$ is algebraically closed and $\operatorname{dim}_{\mathbb{F}} \mathcal{D}_{1}<\infty$. This also implies $\operatorname{dim} \mathcal{D}_{g}=1$, for all $g \in T$. Let $\left\{X_{u} \mid u \in T\right\}$ be a homogeneous basis of $\mathcal{D}$. Then $X_{u} X_{v}=\sigma(u, v) X_{u v}$, for some $\sigma(u, v) \in \mathbb{F}^{\times}$. Since $\mathcal{D}$ is associative, from $\left(X_{u} X_{v}\right) X_{w}=X_{u}\left(X_{v} X_{w}\right)$, we derive that $\sigma$ is a 2-cocycle. Hence, $\mathcal{D} \cong \mathbb{F}^{\sigma} T$, the twisted group algebra of $T$ by $\sigma$. Conversely, for any finite group $T$ and any $\sigma \in Z^{2}\left(T, \mathbb{F}^{\times}\right)$, the natural $T$-grading on $\mathbb{F}^{\sigma} T$ turns it into a graded division algebra.

Now, assume that $T$ is abelian. Let $\beta(u, v)=\sigma(u, v) \sigma(v, u)^{-1}$. A direct computation shows that $\beta$ is an alternating bicharacter; moreover, $\mathcal{D}$ is central if and only if $\beta$ is nondegenerate. Finally, Theorem 2.15 of [9] tells that the pair $(T, \beta)$ uniquely determines an isomorphism class of finite-dimensional central graded division algebras over $\mathbb{F}$ with commutative support. Hence, if $T$ is abelian, the pairs $(T, \beta)$ are in bijection with the elements of the second cohomology group $H^{2}\left(T, \mathbb{F}^{\times}\right)$. 
2.4. Realization of graded division algebras with commutative support. Let $\varepsilon$ be a primitive $n$-root of unity. Consider the elements

$$
X=\left(\begin{array}{cccccc}
0 & 1 & 0 & \cdots & 0 & 0 \\
0 & 0 & 1 & \cdots & 0 & 0 \\
\vdots & \vdots & \vdots & & \vdots & \vdots \\
0 & 0 & 0 & \cdots & 0 & 1 \\
1 & 0 & 0 & \cdots & 0 & 0
\end{array}\right), \quad Y=\left(\begin{array}{ccccc}
\varepsilon^{n-1} & & & & 0 \\
& \varepsilon^{n-2} & & & \\
& & \ddots & & \\
& & & \varepsilon & \\
0 & & & & 1
\end{array}\right) .
$$

Note that $\varepsilon X Y=Y X$ and $X^{n}=Y^{n}=1$. Moreover, $\left\{X^{i} Y^{j} \mid i, j=0,1, \ldots, n\right\}$ is a vector space basis of $M_{n}(\mathbb{F})$. Also, $\mathcal{A}_{(i, j)}=\operatorname{Span}\left\{X^{i} Y^{j}\right\}$ constitute a $\mathbb{Z}_{n} \times \mathbb{Z}_{n^{-}}$ grading on $M_{n}(\mathbb{F})$. This grading is called $\varepsilon$-grading, and it is a division grading.

Now, if $M_{n}(\mathbb{F})$ is endowed with a division grading, then, as mentioned in the previous section, the support $T$ of the grading is a group, and the product is determined by a non-degenerate alternating bicharacter $\beta: T \times T \rightarrow \mathbb{F}^{\times}$. Thus, we obtain a decomposition $T=H_{1}^{2} \times H_{2}^{2} \times \cdots \times H_{s}^{2}$, where each $H_{i}$ is $\beta$-invariant and $H_{i} \cong \mathbb{Z}_{m_{i}}$. Moreover, we obtain

$$
M_{n}=M_{m_{1}} \otimes M_{m_{2}} \otimes \cdots \otimes M_{m_{s}}
$$

where Supp $M_{m_{i}}=H_{i}^{2}$ and $M_{m_{i}}$ has an $\varepsilon_{i}$-grading (see [9, Section 2.2] for more details).

Thus, if $\mathcal{D}$ is a central finite-dimensional graded division algebra over an algebraically closed field $\mathbb{F}$, then we can realize $\mathcal{D}$ as a matrix algebra. Such realization

is made after a choice of Kronecker product identification as in (3), and, for each $M_{m_{i}}$, a choice of a basis as in (2).

\section{Degree-INVERTING INVOLUtion ON GRADED Division ALGEBRAS}

As mentioned above, over an algebraically closed field $\mathbb{F}$, a finite-dimensional $G$ graded-division algebra assumes the form $\mathbb{F}^{\sigma} T$, where $T \subseteq G$ is a finite subgroup, and $\sigma: T \times T \rightarrow \mathbb{F}^{\times}$is a 2-cocycle.

Lemma 3. Given $\sigma \in Z^{2}\left(T, \mathbb{F}^{\times}\right)$, let $\bar{\sigma}: T \times T \rightarrow \mathbb{F}^{\times}$be defined by $\bar{\sigma}(u, v)=$ $\sigma\left(v^{-1}, u^{-1}\right)$. Then $[\bar{\sigma}]=\left[\sigma^{-1}\right]$.

Proof. We have

$$
\begin{aligned}
\sigma(u, v) \bar{\sigma}(u, v) & =\sigma(u, v) \sigma\left(v^{-1}, u^{-1}\right) \\
& =\sigma\left(u v, v^{-1}\right)^{-1} \sigma\left(v, v^{-1}\right) \sigma\left(u, v v^{-1}\right) \sigma\left(v^{-1}, u^{-1}\right) .
\end{aligned}
$$

Also,

$$
\sigma\left(u v, v^{-1}\right)=\sigma\left(u v v^{-1}, u^{-1}\right)^{-1} \sigma\left(v^{-1}, u^{-1}\right) \sigma\left(u v, v^{-1} u^{-1}\right) .
$$

Thus, continuing from the first equation,

$$
\begin{aligned}
\sigma(u, v) \sigma(\bar{u}, v) & =\sigma\left(u, u^{-1}\right) \sigma\left(v, v^{-1}\right) \sigma\left(u v,(u v)^{-1}\right)^{-1} \\
& =\delta \lambda(u, v),
\end{aligned}
$$

where $\lambda(u):=\sigma\left(u, u^{-1}\right)$.

We fix a $\sigma \in Z^{2}\left(T, \mathbb{F}^{\times}\right)$, and a homogeneous basis $\left\{X_{u} \mid u \in T\right\}$ of $\mathbb{F}^{\sigma} T$.

Proposition 4. $\mathbb{F}^{\sigma} T$ admits a degree-inverting involution if and only if $[\sigma]^{2}=1$. 
Proof. Assume that $\rho$ is a degree-inverting involution on $\mathbb{F}^{\sigma} T$. Let $\mu: T \rightarrow \mathbb{F}^{\times}$be such that $\rho\left(X_{u}\right)=\mu(u) X_{u^{-1}}$, for all $u \in T$. Note that, for any $u, v \in T$,

$$
\begin{aligned}
& \rho\left(X_{u} X_{v}\right)=\rho\left(X_{v}\right) \rho\left(X_{u}\right)=\mu(u) \mu(v) \sigma\left(v^{-1}, u^{-1}\right) X_{v^{-1} u^{-1}} \\
& \rho\left(X_{u} X_{v}\right)=\sigma(u, v) \rho\left(X_{u v}\right)=\sigma(u, v) \mu(u v) X_{(u v)^{-1}} .
\end{aligned}
$$

Thus $\sigma=(\delta \mu) \bar{\sigma}$, which implies $[\sigma]^{2}=1$, by Lemma 3 ,

Conversely, if $[\sigma]=\left[\sigma^{-1}\right]=[\bar{\sigma}]$, let $\mu: T \rightarrow \mathbb{F}^{\times}$be such that $\sigma=(\delta \mu) \bar{\sigma}$. We claim that $\rho: \mathbb{F}^{\sigma} T \rightarrow \mathbb{F}^{\sigma} T$ defined by $\rho\left(X_{u}\right)=\mu(u) X_{u^{-1}}$ is a degree-inverting involution. By definition, $\rho$ inverts the degrees, so we only need to show that it is an involution. We have

$$
\begin{aligned}
& \rho\left(X_{u} X_{v}\right)=\sigma(u, v) \mu(u v) X_{(u v)^{-1}}, \\
& \rho\left(X_{v}\right) \rho\left(X_{u}\right)=\mu(v) \mu(u) \sigma\left(v^{-1}, u^{-1}\right) X_{v^{-1} u^{-1}},
\end{aligned}
$$

and both coincide by the choice of $\mu$. Finally,

$$
\rho \rho\left(X_{u}\right)=\mu(u) \mu\left(u^{-1}\right) X_{u} .
$$

So, we need to show that $\mu(u) \mu\left(u^{-1}\right)=1$, for all $u \in T$. However, we note that, for any $u, v \in T$, we have

$$
\frac{\mu(u) \mu(v)}{\mu(u v)}=\frac{\sigma(u, v)}{\bar{\sigma}(u, v)} .
$$

In particular, $\mu(u) \mu\left(u^{-1}\right)=\sigma\left(u, u^{-1}\right) \bar{\sigma}\left(u, u^{-1}\right)^{-1} \mu\left(u u^{-1}\right)=\mu(1)$, for any $u \in T$. Taking $u=1$, we obtain $\mu(1)=1$. Hence, $\mu(u) \mu\left(u^{-1}\right)=1$, for any $u \in T$, and we are done.

Lemma 5. There exists an isomorphism $\operatorname{Aut}_{G}\left(\mathbb{F}^{\sigma} T\right) \cong \operatorname{Hom}\left(T, \mathbb{F}^{\times}\right)$.

Proof. Given $\psi \in \operatorname{Aut}_{G}\left(\mathbb{F}^{\sigma} T\right)$, we have $\psi\left(X_{u}\right)=\chi(u) X_{u}$, for some $\chi: T \rightarrow \mathbb{F}^{\times}$, for all $u \in T$. It is easy to check that $\chi$ is a group homomorphism. Conversely, given $\chi: T \rightarrow \mathbb{F}^{\times}$, the map $\psi$ defined by $\psi\left(X_{u}\right)=\chi(u) X_{u}$ is a $G$-graded automorphism of $\mathbb{F}^{\sigma} T$. So, we obtain a bijection $\psi \mapsto \chi$.

Finally, note that, if $\psi_{i} \mapsto \chi_{i}$, for $i=1,2$, then $\psi_{1} \psi_{2} \mapsto \chi_{1} \chi_{2}$. So, the bijection is a group isomorphism.

Denote $\hat{T}=\operatorname{Hom}\left(T, \mathbb{F}^{\times}\right)$. As a consequence of the previous lemma, $\operatorname{Aut}_{G}\left(\mathbb{F}^{\sigma} T\right) \cong$ $\hat{T}$ is an abelian group.

Lemma 6. Let $\rho$ be a degree-inverting involution on $\mathbb{F}^{\sigma} T$. Then, for any $\psi \in$ $\operatorname{Aut}_{G}\left(\mathbb{F}^{\sigma} T\right), \rho \circ \psi$ is a degree-inverting involution on $\mathbb{F}^{\sigma} T$. Every degree-inverting involution is obtained by such way.

Proof. Using that $\psi\left(X_{u}\right)=\chi(u) X_{u}$, for all $u \in T$, we obtain that $\rho \circ \psi$ is a degreeinverting involution by direct computation. If $\rho^{\prime}$ is another degree-inverting involution, then $\rho \rho^{\prime}$ is a graded automorphism, thus $\rho \rho^{\prime}=\psi$, for some $\psi \in \operatorname{Aut}_{G}\left(\mathbb{F}^{\sigma} T\right)$. Thus, $\rho^{\prime}=\rho \circ \psi$.

Given a group $H$, we denote $S(H)=\left\{h^{2} \mid h \in H\right\}$. Notice that, if $H$ is abelian, then $S(H)$ is a subgroup of $H$.

Lemma 7. $\rho$ and $\rho \circ \psi$ are equivalent if and only if $\psi \in S\left(\operatorname{Aut}_{G}\left(\mathbb{F}^{\sigma} T\right)\right)$. 
Proof. For any $\psi \in \operatorname{Aut}_{G}\left(\mathbb{F}^{\sigma} T\right)$, note that $\rho \circ \psi=\psi^{-1} \circ \rho$.

So, if $\psi=\varphi^{2}$, for some $\varphi \in \operatorname{Aut}_{G}\left(\mathbb{F}^{\sigma} T\right)$, then

$$
\rho \psi=\rho \varphi \varphi=\varphi^{-1} \rho \varphi,
$$

which shows that $\rho \psi \sim \rho$. Conversely, assume that $\rho \psi=\varphi^{-1} \rho \varphi$, for some $\varphi$. Then we obtain $\rho \psi=\rho \varphi^{2}$, which implies $\psi=\varphi^{2} \in S\left(\operatorname{Aut}_{G}\left(\mathbb{F}^{\sigma} T\right)\right)$.

We summarize the results.

Theorem 8. Let $\mathbb{F}$ be a field, $T$ a finite group, and $\sigma: T \times T \rightarrow \mathbb{F}^{\times}$a 2-cocycle. Then $\mathbb{F}^{\sigma} T$ admits a degree-inverting involution if and only if $[\sigma]^{2}=1$. In this case, there exist $|\hat{T} / S(\hat{T})|$ non-equivalent classes of degree-inverting involution on $\mathbb{F}^{\sigma} T$.

Now, we are interested in the case where we have simultaneously $\mathbb{F}^{\sigma} T$ isomorphic to a matrix algebra, and $[\sigma]$ of order 2 . The last one can be achieved if we compute the Schur multiplier $M(T)$. The former one is equivalent to: (a) $|T|=n^{2}$, for some $n$, and (b) $T$ admits an irreducible (projective) $\sigma$-representation of degree $n$.

Although some works were dedicated to either answer the first question, or to compute the Schur multiplier (see, for instance, [15, 16]), we were not able to find a single example of a non-abelian group satisfying both conditions. So we leave the following question.

Question. Find a non-abelian finite group $T$ of order $n^{2}$, for some $n \in \mathbb{N}$, and a 2-cocyle $\sigma: T \times T \rightarrow \mathbb{F}^{\times}$such that $[\sigma]^{2}=1$, and $\mathbb{F}^{\sigma} T \cong M_{n}(\mathbb{F})$.

3.1. Abelian case. Things become easier if we assume a priori the grading group abelian.

The following was essentially proved in [11]:

Lemma 9. Let $\psi_{0}: \mathcal{D} \rightarrow \mathcal{D}$ be a degree-inverting anti-automorphism, where $\mathcal{D}$ is a central finite-dimensional graded division algebra with support $T$, where $T$ is an abelian group. Then $T$ is an elementary 2-group.

Proof. As mentioned in Subsection 2.4. Supp $\mathcal{D}=H_{1}^{2} \times H_{2}^{2} \times \cdots \times H_{s}^{2}$, where each $H_{i} \cong \mathbb{Z}_{n_{i}}$, and $\mathcal{D} \cong M_{n_{1}} \otimes M_{n_{2}} \otimes \cdots \otimes M_{n_{s}}$, where each $M_{n_{i}}$ is endowed with an $\varepsilon_{i}$-grading.

Since every nonzero homogeneous component of $\mathcal{D}$ has dimension 1 , we see that each $1 \otimes \cdots 1 \otimes M_{n_{i}} \otimes 1 \cdots \otimes 1$ is invariant under the anti-automorphism, with support $1 \times \cdots 1 \times H_{i}^{2} \times 1 \cdots \times 1$. From Lemma 4.6 of [11], we obtain $n_{i}=2$ and $H_{i} \cong \mathbb{Z}_{2}$.

So, an immediate consequence is the following remark:

Corollary 10. Let $\mathcal{D}$ be a central finite-dimensional graded division algebra over an algebraically closed field $\mathbb{F}$, and assume that $\operatorname{Supp} \mathcal{D}$ is commutative. Then an involution on $\mathcal{D}$ is a degree-preserving involution if and only if it is a degreeinverting involution.

\section{Degree-InVERting involution on matrix Algebras}

In this section we investigate degree-inverting involution on matrix algebras over an algebraically closed field. The arguments in this section are a copy of the ordinary case [9, Section 2.4] (see also the original paper by Elduque [10]). If a matrix 
algebra is endowed with a grading and a degree-inverting involution, then its support does not need to be commutative. This is a contrast with the degree-preserving involution case (see, for instance, [9, Proposition 2.49]).

We fix an algebraically closed field $\mathbb{F}$ and an arbitrary group $G$. Let $\mathcal{D}$ be a finite-dimensional $G$-graded division algebra, and let $T$ be its support (then $T \subseteq G$ is a finite subgroup). Let $V$ be a finite-dimensional $G$-graded right $\mathcal{D}$-module. We define

$$
V^{*}=\{f: V \rightarrow \mathcal{D}, f \text { is a graded } \mathcal{D} \text {-linear map }\} .
$$

Thus, $V^{*}$ has a natural $G$-grading. For homogeneous $f \in V^{*}$ and $v \in V$, we denote $\langle f, v\rangle=f(v)$ to emphasize the duality between $V$ and $V^{*}$. Moreover, one has

$$
\operatorname{deg}\langle f, v\rangle=\operatorname{deg} f \operatorname{deg} v .
$$

Let $\mathcal{R}=\operatorname{End}_{\mathcal{D}}(V)$. Then $\mathcal{R}$ is a matrix algebra endowed with a $G$-grading. The natural action of $\mathcal{R}$ on $V$ turns $V$ a graded left $\mathcal{R}$-module. Also, $V^{*}$ has a structure of graded right $\mathcal{R}$-module given by

$$
\langle f r, v\rangle=\langle f, r v\rangle, \quad r \in \mathcal{R}, f \in V^{*}, v \in V .
$$

Assume that $\mathcal{R}$ has a degree-inverting anti-automorphism $\psi$. Then $V^{*}$ becomes a left $\mathcal{R}$-module by

$$
r \cdot f:=f \psi(r), \quad r \in \mathcal{R}, f \in V^{*} .
$$

Lemma 11. With (4), $V^{*}$ is an inverted-graded left $\mathcal{R}$-module, that is, $V^{*}$ is a left $\mathcal{R}$-module and

$$
\mathcal{R}_{g} \cdot V_{t}^{*} \subseteq V_{t g^{-1}}^{*}, \quad \forall g, t \in G .
$$

Proof. Let $r \in \mathcal{R}_{g}, f \in V_{t}^{*}, v \in V_{h}$. Then

$$
\operatorname{deg}(r \cdot f) h=\operatorname{deg}\langle r \cdot f, v\rangle=\operatorname{deg}\langle f \psi(r), v\rangle=\operatorname{deg}\langle f, \psi(r) v\rangle=t g^{-1} h,
$$

thus, $\mathcal{R}_{g} V_{t}^{*} \subseteq V_{t g^{-1}}^{*}$.

For any $G$-graded vector space $W=\bigoplus_{g \in G} W_{g}$, we define $W^{[-]}=\bigoplus_{g \in G} W_{g}^{[-]}$, where $W_{g}^{[-]}=W_{g^{-1}}$. These are known as Veronese modules (see 12, Example 1.2.7], for a more general construction).

Lemma 12. $V$ is an inverted-graded left $\mathcal{R}$-module if and only if $V^{[-]}$is a graded left $\mathcal{R}$-module.

Proof. Assume that $V^{[-]}$is a graded left $\mathcal{R}$-module. Then

$$
\mathcal{R}_{g} V_{t}=\mathcal{R}_{g} V_{t^{-1}}^{[-]} \subseteq V_{g t^{-1}}^{[-]}=V_{t g^{-1}} .
$$

Conversely, if $V$ is an inverted-graded left $\mathcal{R}$-module, then

$$
\mathcal{R}_{g} V_{t}^{[-]}=\mathcal{R}_{g} V_{t^{-1}} \subseteq V_{t^{-1} g^{-1}}=V_{g t}^{[-]} .
$$

Lemma 13. There exists a degree-inverting $\mathcal{R}$-isomorphism $\varphi_{1}: V^{\left[g_{0}\right]} \rightarrow V^{*}$, for some $g_{0} \in G$. Equivalently, $\varphi_{1}: V^{\left[g_{0}\right]} \rightarrow V^{*[-]}$ is a G-graded $\mathcal{R}$-isomorphism.

Proof. It follows from Lemma 12 and Lemma 2.7 of $[9]$.

From now on, we fix $g_{0} \in G$ and $\varphi_{1}: V^{\left[g_{0}\right]} \rightarrow V^{*}$, as in Lemma 13 . 
Lemma 14. There exists a homogeneous anti-automorphism $\psi_{0}: \mathcal{D} \rightarrow \mathcal{D}$ such that

$$
\varphi_{1}(v d)=\psi_{0}(d) \varphi_{1}(v)
$$

for all $v \in V, d \in \mathcal{D}$. Moreover, $\operatorname{deg} \psi_{0}(d)=g_{0}^{-1}(\operatorname{deg} d)^{-1} g_{0}$, for any nonzero homogeneous $d \in D$.

Proof. For any homogeneous $d \in \mathcal{D}$, let $R_{d}: V \rightarrow V$ be the right multiplication by $d$, and $L_{d}: V^{*} \rightarrow V^{*}$ the left multiplication. We will prove that the following sets coincide:

$$
\begin{aligned}
& S_{1}=\left\{\varphi: V^{[g]} \rightarrow V^{*} \text { degree-inverting } \mathcal{R} \text {-isomorphism, for some } g \in G\right\}, \\
& S_{2}=\left\{\varphi_{1} \circ R_{d} \mid d \in \mathcal{D}^{\times} \text {homogeneous }\right\}, \\
& S_{3}=\left\{L_{d} \circ \varphi_{1} \mid d \in \mathcal{D}^{\times} \text {homogeneous }\right\} .
\end{aligned}
$$

It is clear that $S_{2}, S_{3} \subseteq S_{1}$. Given $\varphi \in S_{1}$, we have $\varphi_{1}^{-1} \circ \varphi \in \operatorname{End}_{\mathcal{R}}(V) \cong \mathcal{D}$. Thus, for some nonzero homogeneous $d \in \mathcal{D}$, we have $\varphi_{1}^{-1} \circ \varphi=R_{d}$; which implies $\varphi=\varphi_{1} \circ R_{d} \in S_{2}$. Similarly, $\varphi \circ \varphi_{1}^{-1} \in \operatorname{End}_{\mathcal{R}}\left(V^{*}\right) \cong \mathcal{D}$, so we can find a nonzero homogeneous $d \in \mathcal{D}$ such that $\varphi \circ \varphi_{1}^{-1}=L_{d}$. Hence, $\varphi=L_{d} \circ \varphi_{1} \in S_{3}$.

Now, since $S_{2}=S_{3}$, given a nonzero homogeneous $d \in \mathcal{D}$, we can find a nonzero homogeneous $d^{\prime} \in \mathcal{D}$ such that $L_{d^{\prime}} \circ \varphi_{1}=\varphi_{1} \circ R_{d}$. Define $\psi_{0}: \mathcal{D} \rightarrow \mathcal{D}$ linearly, such that $\psi(d)=d^{\prime}$. By construction, $\psi_{0}$ is a linear isomorphism, and it is an antihomomorphism. Also, $L_{\psi(d)} \circ \varphi_{1}=\varphi_{1} \circ R_{d}$ is equivalent to $\psi(d) \varphi_{1}(v)=\varphi_{1}(v d)$, for all $v \in V$. Moreover, from this relation, we derive the following:

$$
\operatorname{deg} \psi(d)\left((\operatorname{deg} v) g_{0}\right)^{-1}=\left((\operatorname{deg} v)(\operatorname{deg} d) g_{0}\right)^{-1} .
$$

Or, equivalently, $\operatorname{deg} \psi(d)=g_{0}^{-1}(\operatorname{deg} d)^{-1} g_{0}$.

Remark 15. If it happens that $g_{0} \in \operatorname{Supp} \mathcal{D}$, then, by the proof of Lemma 14 we can replace $\varphi_{1}$ by $\varphi_{1} \circ R_{d_{0}}$, where $d_{0} \in \mathcal{D}$ is homogeneous with $\operatorname{deg} d_{0}=g_{0}$. Thus, $\operatorname{deg} \psi_{0}(d)=(\operatorname{deg} d)^{-1}$ for all homogeneous $d$, so that the new $\psi_{0}: \mathcal{D} \rightarrow \mathcal{D}$ is a degree-inverting involution on $\mathcal{D}$.

Now, we have a non-degenerate $\mathbb{F}$-bilinear form $B: V \times V \rightarrow \mathcal{D}$ given by

$$
B(v, w)=\left\langle\varphi_{1}(v), w\right\rangle .
$$

This form satisfies the following properties:

(i) $\operatorname{deg} B(v, w)=g_{0}^{-1}(\operatorname{deg} v)^{-1} \operatorname{deg} w$, for all homogeneous $v, w \in V$,

(ii) $B$ is $\psi_{0}$-sesquilinear, that is, $B(v d, w)=\psi_{0}(d) B(v, w), B(v, w d)=B(v, w) d$, $v, w \in V, d \in \mathcal{D}$

(iii) $B(r v, w)=B(v, \psi(r) w), v, w \in V, r \in \mathcal{R}$.

Conversely, a pair $\left(B, \psi_{0}\right)$ satisfying (i)-(iii) determines uniquely $\psi$, that is, we can recover $\psi$ from the pair $\left(B, \psi_{0}\right)$. Indeed, let $\left\{w_{1}, \ldots, w_{n}\right\}$ be a homogeneous $\mathcal{D}$-basis of $V$. Let $\Phi=\left(x_{i j}\right)$, where $x_{i j}=B\left(w_{i}, w_{j}\right)$, be the matrix of $B$. Given $r \in \mathcal{R}$, let $R=\left(r_{i j}\right)$ be its matrix form, and $\psi(R)=\left(r_{i j}^{\prime}\right)$ the matrix form of $\psi(r)$. Then, we have

$$
\begin{aligned}
& B\left(r w_{k}, w_{\ell}\right)=B\left(\sum_{i=1}^{n} w_{i} r_{i k}, w_{\ell}\right)=\sum_{i=1}^{n} \psi_{0}\left(r_{i k}\right) x_{i \ell} \\
& B\left(w_{k}, \psi(r) w_{\ell}\right)=B\left(w_{k}, \sum_{i=1}^{n} w_{i} r_{i \ell}^{\prime}\right)=\sum_{i=1}^{n} x_{k i} r_{i \ell}^{\prime}
\end{aligned}
$$


So, we obtain the equation $\psi_{0}(R)^{t} \Phi=\Phi R$. Hence,

$$
\psi: X \in \mathcal{R} \mapsto \Phi^{-1} \psi_{0}\left(X^{t}\right) \Phi \in \mathcal{R},
$$

where we identify, via Kronecker product, $\mathcal{R}=M_{n}(\mathcal{D}), \psi_{0}(X)$ means that we are applying $\psi_{0}$ in the entries of $X$, and ${ }^{t}$ is the usual matrix transposition of the $n \times n$ matrices $M_{n}(\mathcal{D})$.

We summarize the results obtained so far:

Proposition 16 (cf. 9, Theorem 2.57]). Let $G$ be any group, $\mathcal{D}$ a graded division algebra, $V$ a finite-dimensional graded right $\mathcal{D}$-module and $\mathcal{R}=\operatorname{End}_{\mathcal{D}}(V)$. Assume that $\psi$ is a degree-inverting anti-automorphism of $\mathcal{R}$. Then there exist $g_{0} \in G$, an anti-automorphism $\psi_{0}$ on $\mathcal{D}$ satisfying $\operatorname{deg} \psi_{0}(d)=g_{0}^{-1}(\operatorname{deg} d)^{-1} g_{0}$ for all homogeneous $d \in \mathcal{D}$, and a non-degenerate form $B: V \times V \rightarrow \mathcal{D}$ satisfying (i)-(iii). If $\left(\psi_{0}^{\prime}, B^{\prime}\right)$ is another such pair, then there exists a nonzero homogeneous $d \in \mathcal{D}$ such that $B^{\prime}=d B$ and $\psi_{0}^{\prime}(x)=d \psi_{0}(x), \forall x \in \mathcal{D}$.

Conversely, given a pair $\left(\psi_{0}, B\right)$ satisfying $(i)-($ iii $)$, there exists a degree-inverting anti-automorphism on $\mathcal{R}$.

Now, from now on, we assume that $\psi$ is a degree-inverting involution, that is, $\psi^{2}=1$.

Lemma 17. If $\psi$ is an involution, then

$$
B(w, v)=\varepsilon_{B} \psi_{0}(B(v, w)), \quad \forall v, w \in V
$$

where $\varepsilon_{B} \in\{1,-1\}$.

Proof. Define $\bar{B}(v, w)=\psi_{0}(B(w, v))$. Then $\bar{B}$ is a non-degenerate $\psi_{0}$-sesquilinear form satisfying (ii). Thus, we can find an invertible $\mathcal{D}$-linear $Q: V \rightarrow V$ such that $\bar{B}(v, w)=B(Q v, w)$, for all $v, w \in V$. Hence, for any $r \in \mathcal{R}, v, w \in V$,

$$
\begin{aligned}
B(v, r w) & =B(\psi(r) v, w)=\psi_{0} \bar{B}(w, \psi(r) v)=\psi_{0} B(Q w, \psi(r) v)=\psi_{0} B(r Q w, v)= \\
& =\bar{B}(v, r Q w)=B(Q v, r Q w) .
\end{aligned}
$$

Taking $r=1$, we see that $B(v, w)=B(Q v, Q w)$ for all $v, w \in V$. Hence, we have

$$
B(v, r w)=B(Q v, r Q w)=B\left(v, Q^{-1} r Q w\right) .
$$

So $r=Q^{-1} r Q$, for all $r \in \mathcal{R}$. This gives $Q=\lambda \in \mathbb{F}$. Moreover, $B(v, w)=$ $\lambda^{2} B(v, w)$, for all $v, w \in V$, which implies $\lambda \in\{1,-1\}$. Thus, $\psi_{0} B(w, v)=$ $\bar{B}(v, w)=\varepsilon_{B} B(v, w)$, where $\varepsilon_{B}=\lambda$.

As a result, $B$ is balanced, that is, $B(v, w)=0$ if and only if $B(w, v)=0$.

Given any $\mathcal{D}$-subspace $U \subseteq V$, we define

$$
U^{\perp}=\{x \in V \mid B(x, U)=0\}=\{x \in V \mid B(U, x)=0\} .
$$

The following result is standard:

Lemma 18. Let $B: V \times V \rightarrow \mathcal{D}$ be a non-degenerate balanced $\mathbb{F}$-bilinear form. Given a D-subspace $U \subseteq V$, we have $V=U \oplus U^{\perp}$ if and only if $\left.B\right|_{U}$ is nondegenerate.

Now, using Lemma 18. we can construct a homogeneous $\mathcal{D}$-basis of $V$

$$
\left\{v_{1}, \ldots, v_{m}, v_{m+1}^{\prime}, v_{m+1}^{\prime \prime}, \ldots, v_{s}^{\prime}, v_{s}^{\prime \prime}\right\}
$$

satisfying 
(a) $B\left(v_{i}, v_{i}\right) \neq 0, i=1,2, \ldots, m$,

(b) $B\left(v_{j}^{\prime}, v_{j}^{\prime \prime}\right)=1, j>m$,

(c) all the remaining $B(v, w)=0$.

Let $g_{i}=\operatorname{deg} v_{i}, g_{j}^{\prime}=\operatorname{deg} v_{j}^{\prime}, g_{j}^{\prime \prime}=\operatorname{deg} v_{j}^{\prime \prime}$. If $m>0$, then $T \ni \operatorname{deg} B\left(v_{1}, v_{1}\right)=g_{0}^{-1}$. Also,

$$
1=\operatorname{deg} B\left(v_{j}^{\prime}, v_{j}^{\prime \prime}\right)=g_{0}^{-1} g_{j}^{\prime-1} g_{j}^{\prime \prime},
$$

so $g_{j}^{\prime \prime}=g_{j}^{\prime} g_{0}$, for all $j>m$. Moreover, we have

Lemma 19. If $s>m$, then $g_{0}^{2}=1$.

Proof. Since

$$
B\left(v_{s}^{\prime \prime}, v_{s}^{\prime}\right)=\varepsilon_{B} \psi_{0}\left(B\left(v_{s}^{\prime}, v_{s}^{\prime \prime}\right)\right)=\varepsilon_{B} 1,
$$

we obtain $1=g_{0}^{-1}\left(g^{\prime \prime}\right)^{-1} g^{\prime}=g_{0}^{-2}$. Thus, $g_{0}^{2}=1$.

Now, if $\varepsilon_{B}=1$ then we call $\psi$ orthogonal, and otherwise, $\psi$ is symplectic. We note that $\varepsilon_{B}=-1$ implies $m=0$ in the previous notations. Using (6), we can construct the matrix of $\Phi$, and determine $\psi$ in matrix form. It will be convenient to use the basis $\left\{v_{1}, \ldots, v_{m}, v_{m+1}^{\prime}, \ldots, v_{s}^{\prime}, v_{m+1}^{\prime \prime}, \ldots, v_{s}^{\prime \prime}\right\}$. We summarize the results

Theorem 20. Let $\mathcal{R}=M_{n}(\mathcal{D})$ be a matrix algebra endowed with a G-grading parametrized by $(\mathcal{D}, \gamma)$. Then $\mathcal{R}$ admits a degree-inverting involution $\psi$ if and only if there exists $g_{0} \in G$, the graded division algebra $\mathcal{D}$ admits an involution $\psi_{0}$ satisfying $\operatorname{deg} \psi_{0}(d)=g_{0}^{-1}(\operatorname{deg} d)^{-1} g_{0}, \forall d \in \mathcal{D}$ homogeneous, and

$$
\gamma=\left(g_{1}, \ldots, g_{m}, g_{m+1}^{\prime}, \ldots, g_{s}^{\prime}, g_{m+1}^{\prime \prime}, \ldots, g_{s}^{\prime \prime}\right)
$$

where $g_{j}^{\prime \prime}=g_{j}^{\prime} g_{0}$, for all $j>m$. Moreover, if $g_{0} \notin T$ then $m=0$; if $g_{0} \in T$, then we can assume $\psi_{0}$ a degree-inverting involution; and if $s>m$ then $g_{0}^{2}=1$.

Let $\left\{X_{u} \mid u \in T\right\}$ be a homogeneous basis of $\mathcal{D}$. In any case, $\psi\left(e_{i j} \otimes X\right)=$ $\Phi^{-1} e_{j i} \otimes \psi_{0}(X) \Phi$, for $e_{i j} \otimes X \in \mathcal{R}$, where $\Phi$ is given by:

(i) if $\psi$ is orthogonal,

$$
\Phi=\left(\begin{array}{ccc}
I_{m} \otimes X_{g_{0}} & & \\
& 0 & I_{s} \otimes X_{1} \\
& I_{s} \otimes X_{1} & 0
\end{array}\right) .
$$

(ii) if $\psi$ is symplectic, then

$$
\Phi=\left(\begin{array}{cc}
0 & I_{s} \\
-I_{s} & 0
\end{array}\right) \otimes X_{1} .
$$

Remark 21. It is worth mentioning that, if $G$ is assumed to be abelian, then we obtain a complete description of degree-inverting involutions on $M_{n}(\mathcal{D})$ : the involution $\psi_{0}$ on $\mathcal{D}$ will be degree-inverting, and we apply Corollary 10 .

\section{DEGREE-INVERTING INVOLUTION ON UPPER TRIANGULAR MATRICES}

In this section we shall classify degree-inverting involution on the algebra of upper triangular matrices. The final result is similar to the degree-preserving involution case [6]. However, in the degree-inverting case, the support of the grading does not need to be commutative. We shall improve the result obtained in [11], since we only impose the restriction $\operatorname{char} \mathbb{F} \neq 2$. 
Let $\mathbb{F}$ be an arbitrary field of characteristic not 2 , and $G$ any group. It is known that every group grading on $U T_{n}$ is elementary [18, that is, every grading admits an isomorphic structure where each matrix unit $e_{i j}$ is homogeneous. Moreover, an isomorphism class of $G$-gradings on $U T_{n}$ is uniquely determined by a sequence $\eta=\left(g_{1}, \ldots, g_{n-1}\right) \in G^{n-1}$, where $\operatorname{deg} e_{i, i+1}=g_{i}$, for $i=1,2, \ldots, n-1$ (see [7, Theorem 2.3]).

From now on, we fix a $G$-grading on $U T_{n}$, given by $\eta=\left(g_{1}, g_{2}, \ldots, g_{n-1}\right)$. Let $J=J\left(U T_{n}\right)$ be the Jacobson radical, which is clearly a graded ideal. We denote by $\tau$ the canonical involution of $U T_{n}$, that is, $\tau\left(e_{i j}\right)=e_{n-j+1, n-i+1}$. Note that $\tau$ is the flip along the secondary diagonal of $M_{n}$.

Let $\rho$ be a degree-inverting involution of $U T_{n}$. Since $\rho\left(J^{m}\right)=J^{m}$, for every $m \geq 1$, we have that $\rho$ is a degree-inverting involution on $J / J^{2}$. Moreover, we know that every automorphism of $U T_{n}$ is inner (see, for instance, [14]); hence, $\rho=\operatorname{Int}(u) \circ \tau$, for some inner automorphism $\operatorname{Int}(u)$ (where $u \in U T_{n}$ is invertible). Thus, $\rho\left(e_{i, i+1}+J^{2}\right)=e_{n-i, n-i+1}+J^{2}$; that is, $\operatorname{deg} e_{i, i+1}=\left(\operatorname{deg} e_{n-i, n-i+1}\right)^{-1}$. This proves

Lemma 22. $\left(U T_{n}, \eta\right)$ admits a degree-inverting involution if, and only if, $g_{i}=$ $g_{n-i+1}^{-1}$ for each $i=1,2, \ldots,\left\lceil\frac{n}{2}\right\rceil$.

Proof. The argument above proves the "only if" part. The "if" part is obvious, since $\tau$ will invert degree, under this condition.

Remark 23. Note that, in contrast with the graded-involution case, the existence of a degree-inverting involution does not imply that the support of the grading is commutative.

Now, assume from now on that $\eta$ satisfies the condition of Lemma 22, It is clear that $\tau$ is a degree-inverting involution in this case. Since we wrote $\rho=\operatorname{Int}(u) \circ \tau$, we note that $\operatorname{Int}(u)$ is a graded automorphism of $U T_{n}$. Thus, $u$ is homogeneous of degree 1. Moreover, since $\rho^{2}=1$, one has $\tau(u)= \pm u$. We note that $\tau(u)=-u$ happens only if $n$ is even. Indeed, if $n=2 m+1$, then $\tau\left(e_{m+1, m+1}\right)=e_{m+1, m+1}$. Since $u$ is invertible, the entry $(m+1, m+1)$ of $u$ must be nonzero; and at the same time, it should coincide with its opposite, a contradiction.

Suppose $n=2 m$, and let $D=\operatorname{diag}(1, \ldots, 1,-1, \ldots,-1)$. The involution $s(x)=$ $D \tau(x) D$ is called the symplectic involution of $U T_{n}$.

Finally, if $n=2 m+1$, then we can multiply $u$ by some scalar (note that, $\operatorname{Int}(u)=\operatorname{Int}(\lambda u)$ ), in such a way that its $m+1$ entry is 1 (this is an important step in the proof of the next lemma, see [6. Lemma 2.4]). Also, if $\tau(u)=-u$, then

$$
\rho(x)=u \tau(x) u^{-1}=u D D \tau(x) D D u^{-1}=\operatorname{Int}(u D)(s(x)) .
$$

In this case, $s(u D)=u D$. So, we can replace $u$ by $u D$ to obtain $s(u)=u$. Hence, in any case, we always obtain the equation

$$
\rho=\operatorname{Int}(u) \circ \rho_{0},
$$

with $\rho_{0}(u)=u$, where $\rho_{0}$ is either $\tau$ or $s$.

Lemma 24. Assume char $\mathbb{F} \neq 2$. Let $u$ be an invertible homogeneous element of degree 1. Let $\rho_{0}$ be either $\tau$ or $s$, in such a way that $\rho_{0}(u)=u$; and if $n=2 m+1$, assume that the entry $(m+1, m+1)$ of $u$ is 1 . Then there exists a homogeneous invertible element $v \in U T_{n}$, of degree 1 , such that $u=v \rho_{0}(v)$. 
Proof. The proof is exactly the construction of the proof of Lemma 2.4 of [6] (see also [11, Lemma 6.9]). As an example, we include here the case $n=2 m$, and $\rho_{0}=\tau$. Write

$$
u=\left(\begin{array}{cc}
X & Z \\
0 & Y
\end{array}\right)
$$

where $X, Y \in U T_{m}$ are invertible, and $Z \in M_{m}$. Then

$$
v=\left(\begin{array}{cc}
\operatorname{Id}_{m} & \frac{1}{2} Z \\
0 & Y
\end{array}\right)
$$

satisfies $u=v \tau(v)$. Moreover, let $\mathcal{U}$ be the set of pairs $(i, j)$ such that $u=$ $\sum_{(i, j) \in \mathcal{U}} \alpha_{i j} e_{i j}$, for $\alpha_{i j} \neq 0$. Since $u$ is homogeneous of degree 1 , and every matrix unit is homogeneous; $\operatorname{deg} e_{i j}=1$, for all $(i, j) \in \mathcal{U}$. Now, by construction, $v=\sum_{(i, j) \in \mathcal{U}^{\prime}} \beta_{i j} e_{i j}$, for some $\mathcal{U}^{\prime} \subseteq \mathcal{U}$. In particular, $v$ is a linear combination of homogeneous elements of degree 1 . This imply $v$ homogeneous of degree 1.

The proof is similar for the other cases.

As a conclusion, $\rho=\operatorname{Int}(u) \circ \rho_{0}=\operatorname{Int}(v) \circ \operatorname{Int}\left(\rho_{0}(v)\right) \circ \rho_{0}$, where $\rho_{0}$ is either $\tau$ or $s$, and $\rho_{0}(u)=u$. A straightforward argument shows that, in this case, $\rho$ is equivalent to $\rho_{0}$. Indeed, we need to find a graded automorphism $\varphi$ such that $\varphi\left(\rho_{0}(x)\right)=\rho(\varphi(x))$. Taking $\varphi=\operatorname{Int}(v)$, we have

$$
\rho(\varphi(x))=\rho\left(v x v^{-1}\right)=\operatorname{Int}(v) \operatorname{Int}\left(\rho_{0}(v)\right) \rho_{0}\left(v x v^{-1}\right)=\operatorname{Int}(v)\left(\rho_{0}(x)\right)=\varphi\left(\rho_{0}(x)\right) .
$$

We summarize our main result of this section:

Theorem 25. Let $\mathbb{F}$ be a field of characteristic not 2 , and $G$ any group. Let $\left(U T_{n}, \eta\right)$ be $G$-graded, where $\eta=\left(g_{1}, g_{2}, \ldots, g_{n-1}\right)$. Then $\left(U T_{n}, \eta\right)$ admits a degreeinverting involution if, and only if, $g_{i}=g_{n-i+1}^{-1}$, for all $i=1,2, \ldots, n-1$. In this case, every degree-inverting involution is equivalent either to $\tau$ or to $s$; where $s$ can occur if, and only if, $n$ is even.

Our definition of elementary grading on $U T_{n}$ is not the standard one. Usually one defines an elementary grading on $U T_{n}$ as we did for matrix algebras, that is, a sequence $\gamma=\left(h_{1}, h_{2}, \ldots, h_{n}\right) \in G^{n}$ defines a $G$-grading on $U T_{n}$ by $\operatorname{deg} e_{i j}=h_{i} h_{j}^{-1}$. However, we cannot find a friendly way to write the condition of existence of a degree-inverting involution on $U T_{n}$ in the standard notation. Nonetheless, if the grading group is abelian then the condition is nicely written, and we reobtain a result of [11]:

Corollary 26 ([11, Corollary 5.11]). Let $\mathbb{F}$ be a field of characteristic not 2, and $G$ be an abelian group. Let $U T_{n}$ be endowed with an elementary $G$-grading given by $\gamma=\left(h_{1}, \ldots, h_{n}\right)$. Then $U T_{n}$ admits a degree-inverting involution if and only if $h_{1} h_{n}^{-1}=h_{2} h_{n-1}^{-1}=\cdots=h_{n} h_{1}^{-1}$. In this case, every degree-inverting involution is equivalent either to $\tau$ or to $s$; where $s$ can occur if, and only if, $n$ is even.

\section{REFERENCES}

[1] Y. Bahturin, M. Kochetov, Classification of group gradings on simple Lie algebras of types $\mathcal{A}, \mathcal{B}, \mathcal{C}$ and $\mathcal{D}$, Journal of Algebra, 324 (2010), 2971-2989.

[2] Y. Bahturin, S. Sehgal, M. Zaicev, Group Gradings on Associative Algebras, Journal of Algebra 241 (2001), 677-698.

[3] Y. Bahturin, I. Shestakov, M. Zaicev, Gradings on simple Jordan and Lie algebras, Journal of Algebra 283 (2005), 849-868. 
[4] Y. Bahturin, M. Zaicev, Group Gradings on Matrix Algebras, Canadian Mathematical Bulletin 45 (2002), 499-508.

[5] Y. Bahturin, M. Zaicev, Involutions on graded matrix algebras, Journal of Algebra 315 (2007), $527-540$.

[6] O. Di Vincenzo, P. Koshlukov, R. La Scala, Involutions for upper triangular matrix algebras, Advances in Applied Mathematics 37 (2006), 541-568.

[7] O. Di Vincenzo, P. Koshlukov, A. Valenti, Gradings on the algebra of upper triangular matrices and their graded identities, Journal of Algebra 275 (2004), 550-566.

[8] C. Draper, C. Martn, Gradings on $\mathfrak{g}_{2}$, Linear Algebra and its Applications 418 (2006), 85111.

[9] A. Elduque, M. Kochetov, Gradings on simple Lie algebras, Mathematical Surveys and Monographs, 189. American Mathematical Society (2013).

[10] A. Elduque, Fine gradings on simple classical Lie algebras, Journal of Algebra 324 (2010), $3532-3571$.

[11] L. F. Fonseca, T. C. de Mello, Degree-inverting involutions on matrix algebras, Linear and Multilinear Algebra 66, 1104-1120.

[12] R. Hazrat, Graded Rings and Graded Grothendieck Groups, London Mathematical Society. Lecture Note Ser. 435, Cambridge University Press, Cambridge, 2016.

[13] R. Hazrat, L. Vaš, K-theory classification of graded ultramatricial algebras with involution, Forum Mathematicum 31 (2019), 419-463.

[14] S. Jondrup, Automorphisms and derivations of upper triangular matrix rings, Linear Algebra and its Applications 221 (1995), 205-218.

[15] G. Karpilovsky, Projective representations of finite groups. Monographs and Textbooks in Pure and Applied Mathematics, 94. Marcel Dekker, Inc., New York, 1985.

[16] G. Karpilovsky, The Schur multiplier. London Mathematical Society Monographs. New Series, 2. The Clarendon Press, Oxford University Press, New York, 1987.

[17] J. Patera, H. Zassenhaus, On Lie gradings. I, Linear Algebra and its Applications 112 (1989), $87-159$.

[18] A. Valenti, M. Zaicev, Group gradings on upper triangular matrices, Archiv der Mathematik 89 (2007), 33-40.

Department of Mathematics, State University of Maringá, Maringá, PR, Brazil.

E-mail address, L. Spada: laisspada2@gmail.com

Department of Mathematics, State University of Maringá, Maringá, PR, Brazil.

E-mail address, E. A. Santulo Jr.: easjunior@uem.br

Department of Mathematics, State University of Maringá, Maringá, PR, Brazil.

E-mail address, F. Yasumura: felipeyukihide@gmail.com 